\title{
Intérêt des éponges de collagène pour prévenir la résorption osseuse alvéolaire post-extractionnelle : revue systématique de la littérature
}

\author{
Thomas Blanchy ${ }^{1,2}$, Joanna Babilotte ${ }^{2,3}$, Mathilde Fénelon ${ }^{1,2,3}$, Jean-Marie Marteau ${ }^{1,2}$, \\ Jean-Christophe Fricain ${ }^{1,2,3}$, Sylvain Catros ${ }^{1,2,3^{*}}$
}

1 CHU de Bordeaux, pôle d'0dontologie et de Santé buccale, F-33000 Bordeaux, France

2 Univ. Bordeaux, Bioingénierie Tissulaire, U1026, F-33000 Bordeaux, France

3 INSERM, Bioingénierie Tissulaire, U1026, F-33000 Bordeaux, France

(Reçu le 11 mars 2016, accepté le 13 juin 2016)

Mots clés : extraction dentaire / éponge de collagène / résorption alvéolaire / préservation de crête / facteur de croissance

Key words: tooth extraction / acellular collagen sponge / alveolar bone loss / ridge preservation / growth factor

\begin{abstract}
Résumé - Introduction : La cicatrisation alvéolaire post-extractionnelle est accompagnée systématiquement d'une résorption osseuse plus ou moins importante selon les sites anatomiques et la pathologie initiale. Cette résorption osseuse peut parfois compromettre la mise en place d'implants dentaires. L'objectif de cette revue systématique de la littérature était d'évaluer si les éponges de collagène permettaient de limiter cette résorption osseuse. Matériels et méthodes : Les bases de données Medline et Pubmed ont été consultées pour la période 1986-2015. Résultats : Les études comparant les éponges de collagène à une cicatrisation naturelle n'ont pas montré de différence significative en ce qui concerne la cicatrisation alvéolaire. En revanche, les études comparant l'implantation d'une éponge de collagène comme vecteur de facteurs de croissance à une éponge de collagène seule montraient un effet positif des facteurs de croissance pour corriger un défaut osseux parodontal ou pour limiter la résorption post-extractionnelle. Discussion : Il n'y a pas d'intérêt à placer une éponge de collagène seule dans une alvéole d'extraction pour limiter la résorption osseuse. En revanche, la mise en place dans l'alvéole d'une éponge de collagène associée à des facteurs de croissance permet de maintenir la hauteur et la largeur de la crête alvéolaire. Conclusion : Les éponges de collagène doivent être utilisées uniquement comme des hémostatiques locaux après une extraction dentaire. Rien n'indique dans la littérature que ces éponges pourraient avoir un rôle dans le maintien du volume osseux alvéolaire.
\end{abstract}

\begin{abstract}
Use of acellular collagen sponges to prevent post-extraction alveolar bone resorption: systematic review of the literature. Introduction: Alveolar bone healing following dental extraction is usually accompanied by a resorption of alveolar bone. This resorption of alveolar bone may hinder the insertion of dental implants. The aim of this literature review was to evaluate whether acellular collagen sponge (ACS) grafting could limit alveolar bone resorption and maintain alveolar bone volume after dental extraction. Materials and Methods: The Medline and Pubmed databases were searched for the period 1986-2015. Results: Studies comparing ACS grafting with natural healing did not show any significant differences concerning alveolar bone healing and bone volume preservation. Experiments comparing the implantation of ACS alone versus ACS loaded with growth factors such as rh-BMP2 showed better results using rh-BMP2 for guided periodontal regeneration and post-extraction alveolar bone volume preservation. Discussion: This systematic review of the literature shows that there is no evidence that grafting a collagen sponge inside an alveolar socket would limit alveolar bone resorption. On the other hand, the insertion of a collagen sponge loaded with growth factors (such as rhBMP2) allows maintaining the height and width of the alveolar ridge. Conclusion: Acellular collagen sponges must be used as a local haemostatic agent. They have no effect to maintain alveolar bone volume after dental extraction.
\end{abstract}

* Correspondance : sylvain.catros@u-bordeaux.fr

Note au lecteur : Une erreur s'est glissée dans la version initiale de cet article dans la conclusion de son résumé. Cette nouvelle version publiée le 23 décembre 2016 contient la bonne version de la conclusion du résumé. / Note to the reader: An error has been produced in the initial version of this article in the conclusion of its abstract. This new version published on 23th december 2016 contains the correct conclusion of the abstract. 


\section{Introduction}

L'avulsion dentaire est un acte quotidien en chirurgie orale et la cicatrisation post-extractionnelle débute immédiatement par la formation d'un caillot sanguin dans l'alvéole. Ce caillot est rapidement colonisé par des fibroblastes et des polynucléaires, puis dans un second temps, un tissu de granulation va prendre la place du caillot pendant que les ostéoblastes synthétisent un tissu ostéoïde depuis le fond de l'alvéole. Le tissu de granulation va progressivement se transformer en tissu conjonctif fibreux pendant que la formation osseuse se poursuit avec l'apparition des trabécules minéralisées dès la $3^{e}$ semaine suivant l'extraction dentaire. À partir de la $15^{\mathrm{e}}$ semaine l'alvéole est comblée par un tissu osseux minéralisé même si l'organisation de l'architecture osseuse n'est pas complète avant 24 semaines [1-3].

Cette cicatrisation alvéolaire entraîne une résorption systématique des crêtes alvéolaires dans les premiers mois suivant l'extraction. Cette résorption est plus marquée en vestibulaire qu'en lingual [4]. Elle varie selon les individus et les sites impliqués [5]. Ainsi, une revue systématique de la littérature récente incluant 20 études a montré une résorption osseuse verticale moyenne de $11 \%$ à $22 \%(1,24 \mathrm{~mm} \pm 0,11 \mathrm{~mm}$ en vestibulaire; $0,84 \mathrm{~mm} \pm 0,62 \mathrm{~mm}$ en mésial et $0,80 \mathrm{~mm} \pm$ $0,71 \mathrm{~mm}$ en distal) et une perte horizontale moyenne de $29 \%$ à $63 \%(3,79 \mathrm{~mm} \pm 0,23 \mathrm{~mm})$ après $6-7$ mois [6].

Il faut garder aussi à l'esprit que la résorption osseuse est dépendante de facteurs locaux. La méthode d'extraction est un facteur très important de réduction de la résorption alvéolaire. Il est donc préconisé de réaliser une extraction la plus atraumatique possible [7]. La résorption est également plus faible en absence de lambeau car le périoste amène la vascularisation nécessaire à la cicatrisation $[8,9]$.

$D$ 'après Weng $D$ et al., les méthodes de préservation de crête permettraient de diminuer la lyse osseuse alvéolaire significativement dans le sens horizontal et dans le sens vertical [10]. Cependant, aucun des matériaux de greffe utilisés actuellement ne permettent de préserver complètement le volume osseux après une extraction dentaire et il n'existe pas de consensus ni de recommandations pour utiliser une technique ou un matériau particulier $[8,11]$. Plusieurs techniques chirurgicales de régénération osseuse guidée peuvent donc être utilisées pour limiter cette résorption et elles sont habituellement basées sur l'usage de substituts osseux, allogreffes ou xénogreffes et sur la mise en place de membranes résorbables ou non [12]. Ces méthodes sont coûteuses, parfois complexes à mettre en œuvre et leur efficacité est parfois discutée [13].

L'implantation d'éponges de collagène à visée hémostatique est une pratique courante après une extraction dentaire. L'indication de ces éponges de collagène est de favoriser la formation d'un caillot sanguin stable et de réduire les saignements post-opératoires. Ces éponges sont des dispositifs

\section{Glossaire :}

ACS : Acellular Collagen Sponge (éponge de collagène acellulaire) PCa : Phosphate de Calcium

GBR : Guided Bone Regeneration (régénération osseuse guidée) rhBMP : recombinant human Bone Morphogenetic Protein rhGDF : recombinant human Growth and Differentiation Factor GDF5 : Growth and Differentiation Factor 5

BMP6 : Bone Morphogenetic Protein 6 TCP : Tri-Calcium Phosphate

médicaux, stériles, résorbables, composés de collagène, extraits à partir de derme ou de tendons d'Achille d'origine animale, puis purifiés et séchés. Lors de la formation du caillot sanguin dans l'alvéole l'apport de collagène exogène permet, en augmentant la surface d'adhésion, de promouvoir l'activation des plaquettes, qui aboutit à la formation du clou plaquettaire et participe à l'activation de la coagulation [14].

Les éponges de collagène ont prouvé leur efficacité dans le contrôle de l'hémostase [15]. Selon les recommandations de la Société Française de Chirurgie Orale (SFCO), les extractions dentaires peuvent être effectuées le plus souvent sans modifier le traitement anti-thrombotique du patient. Chez ces patients, la suture des berges de la plaie et la compression sont indispensables après une extraction. Le recours à des hémostatiques locaux, comme l'éponge de collagène, est fortement conseillé. L'arrêt des traitements par anti-thrombotiques avant une extraction dentaire n'est pas justifié dans la plupart des cas [16].

Les éponges de collagène sont des matériaux résorbables, mais peu de fabricants précisent le temps de résorption de leur matériau. Cette vitesse de résorption dépend de l'étendue de l'interface entre l'éponge de collagène et l'alvéole. Selon le rapport de la Haute Autorité de Santé (HAS) sur les agents hémostatiques, cette résorption interviendrait entre 3 et 6 semaines selon les produits [14]. Il y a d'autres utilisations documentées des éponges de collagène en odontologie. En parodontologie, des éponges épaisses et denses sont utilisées comme alternative aux greffes de tissus conjonctifs autologues afin d'augmenter l'épaisseur de gencive kératinisée en chirurgie muco-gingivale, tout en réduisant la morbidité associée aux prélèvements palatins [17]. Par ailleurs, les éponges de collagène ont déjà été utilisées pour délivrer des antibiotiques locaux, notamment la gentamycine [18]. Cependant, l'utilisation d'antibiotiques locaux en odontologie n'est pas recommandée [19].

Par ailleurs, on retrouve une utilisation controversée des éponges de collagène pour préserver le volume d'os alvéolaire après une extraction dentaire. Dans ce cas, ces matériaux sont utilisés de façon empirique dans le but de stabiliser le caillot sanguin, d'éviter l'invagination des tissus mous et d'obtenir une meilleure cicatrisation osseuse. Des auteurs ont proposé d'utiliser des agents hémostatiques pour préserver le volume 
osseux après chirurgie orthopédique [20]. Enfin, les éponges de collagène peuvent servir à délivrer localement des facteurs de croissance, notamment la rhBMP-2 (Infuse ${ }^{\circledR}$, Medtronic). Ce produit n'a pas d'indication en chirurgie dentaire dans le résumé des caractéristiques du produit (RCP) en France, mais depuis 2007 aux États-Unis, la Food and Drug Administration (FDA) a autorisé l'utilisation de ce dispositif dans les comblements sous-sinusiens et les augmentations localisées de crête alvéolaire pour les défauts liés aux alvéoles d'extraction.

L'objectif principal de cette revue systématique de la littérature était donc d'évaluer si les éponges de collagène insérées dans l'alvéole dentaire peuvent permettre de préserver le volume osseux alvéolaire après une extraction dentaire. L'objectif secondaire était d'évaluer comment ces éponges de collagène peuvent être modifiées par l'apport de biomolécules pour favoriser la préservation du volume osseux post-extractionnel.

\section{Matériels et méthodes}

Les bases de données Medline et Pubmed ont été consultées sur la période 1986-2015 pour les articles en anglais avec les mots clés MeSH suivants : «tooth extraction »; « ridge preservation »; « alveolar bone loss »; « collagen/therapeutic $»$; «bone morphogenetic protein 2, recombinant human ». D'autres mots clés ont été utilisés : « absorbable collagen sponge »; « wound healing »; « growth factor»; " bone tissue ». Les références contenues dans les articles identifiés ont également été utilisées au travers d'une recherche manuelle.

Les critères d'inclusions étaient :

- utilisation d'éponges de collagène en comblement osseux post-extractionnel en comparaison à une cicatrisation naturelle et/ou utilisation d'éponges de collagène associées à des biomolécules en comparaison à une cicatrisation avec des éponges de collagène seules ;

- présentation de résultats cliniques et/ou histologiques et/ ou radiologiques de la zone osseuse comblée avant la mise en place d'un implant ;

Les critères d'exclusion des articles étaient :

- revue de littérature ;

- rapport de cas unique (type case report) ;

- études sur le comblement sous-sinusien.

\section{Résultats}

\section{Utilisation des éponges de collagène pour la régénération osseuse intra-orale}

Nous avons retrouvé huit articles où des éponges de collagène ont été mises en place pour préserver l'os alvéolaire après extraction dentaire ou bien pour reconstruire des défauts osseux créés chirurgicalement. Nous n'avons retenu que les études où un groupe témoin sans comblement (cicatrisation naturelle) était également présent. Les groupes traités par les éponges de collagène étaient le plus souvent des groupes contrôles. Ceci nous a permis d'évaluer l'effet des éponges de collagène sur la régénération osseuse.

Parmi les huit articles retenus, nous avons retrouvé six études précliniques et deux études cliniques (Tab. I).

Concernant les expérimentations précliniques, le modèle canin a été utilisé dans 5 études [21-25] alors que des rats ont été utilisés dans 1 autre étude [26]. Dans les six études, des défauts osseux intra-oraux ont été utilisés : dans trois cas il s'agissait de défauts osseux alvéolaires créés chirurgicalement $[21,23,25]$ et dans 3 cas il s'agissait d'alvéoles d'extraction $[22,24,26]$.

Dans les études sélectionnées, la néoformation osseuse après comblement avec une éponge de collagène a été comparée avec un composite rhBMP-2/Collagène [22,25], des défauts laissés vides [21-26], une xénogreffe d'origine bovine [23], ou encore un matériau associant BTCP + Collagène ou du BTCP seul [21].

Dans la majorité des études, on a constaté que la cicatrisation osseuse après extraction dentaire était similaire en présence d'une éponge de collagène ou bien lorsque le défaut était laissé vide [21,22,24,25,27]. Dans un cas, les auteurs ont montré que la cicatrisation osseuse en présence d'une éponge de collagène était retardée lors de la première semaine suivant l'extraction en comparaison avec une cicatrisation naturelle, sans comblement de l'alvéole [26].

Enfin, dans deux études, les auteurs ont observé davantage d'os néoformé dans les alvéoles comblées par des éponges de collagène que dans celles laissées vides. Cependant, les analyses statistiques de ces 2 études sont absentes [23] ou non adaptées [22], ce qui peut nous conduire à relativiser la pertinence de ces résultats.

Dans une étude clinique visant à évaluer l'efficacité d'éponges de collagène imprégnées d'alendronate pour favoriser la régénération osseuse après extraction de dents de sagesse, les groupes contrôles étaient laissés vides ou bien comblés par éponges de collagène sans alendronate. Après 4 mois de cicatrisation, les auteurs ont observé radiographiquement que la résorption osseuse post-extractionnelle était supérieure dans les alvéoles vides par rapport aux alvéoles comblées par éponges de collagène, sans être significative [28].

Dans une étude clinique prospective randomisée en double aveugle incluant 80 patients dont l'objectif principal était d'étudier la néoformation osseuse en présence de rhBMP-2, les groupes contrôles étaient soit des alvéoles d'extraction laissées vides, soit des alvéoles d'extraction comblées par des éponges de collagène. Les résultats ont montré une cicatrisation similaire dans ces deux groupes contrôles, ce qui a démontré qu'il n'y avait pas d'intérêt à placer une éponge de 
Tableau I. Effet des éponges de collagène sur la régénération osseuse intra-orale (rhBMP-2 : protéine morphogénétique osseuse recombinante 2 ; ROG : régénération osseuse guidée ; TCP : phosphate tricalcique ; ZA : acide zolédronique).

Table I. Effect of Acellular Collagen Sponges on intra-oral Bone Regeneration (rhBMP-2: recombinant human Bone Morphogenetic Protein 2; ROG: Guided Bone Regeneration; TCP: TriCalcium Phoshate; ZA: Zoledronic Acid).

\begin{tabular}{|c|c|c|c|c|}
\hline Référence & Objectif de l'étude & Matériel et méthodes & Résultats & $\begin{array}{l}\text { Conclusions (sur les } \\
\text { éponges de collagène) }\end{array}$ \\
\hline $\begin{array}{l}\text { Takahashi et al. } \\
\text { [21] }\end{array}$ & $\begin{array}{l}\text { Test d'éponges colla- } \\
\text { gène / TCP pour la } \\
\text { préservation osseuse } \\
\text { alvéolaire (Olympus } \\
\text { Terumo Biomaterials) }\end{array}$ & $\begin{array}{l}\text { - } 13 \text { chiens (Beagle) } \\
\text { - Défauts osseux maxillaires après } \\
\text { extractions dentaires } \\
-4 \text { groupes : } \\
\text { G1 : comblement avec TCP/ collagène } \\
\text { G2 : comblement avec TCP } \\
\text { G3 : éponge de collagène } \\
\text { G4 : contrôle vide } \\
\text { - Analyse à } 4 \text { ou } 8 \text { semaines }\end{array}$ & $\begin{array}{l}\text { - Volume } 0 \text { s néoformé dans } \\
\text { G1 et G2 significativement } \\
\text { supérieur à G3 et G4 } \\
\text { - Volume } 0 \text { s néoformé dans } \\
\text { G3 et G4 identique }\end{array}$ & $\begin{array}{l}\text { Le volume d'os est similaire } \\
\text { dans le groupe traité par } \\
\text { collagène et dans le groupe } \\
\text { avec alvéoles laissées vides }\end{array}$ \\
\hline $\begin{array}{l}\text { Gerard et al. } \\
\text { [22] }\end{array}$ & \begin{tabular}{|l|} 
- Déterminer l'effet \\
d'une dose de ZA sur \\
la cicatrisation \\
osseuse après extrac- \\
tion dentaire \\
- Évaluer si l'associa- \\
tion rhBMP-2/ACS \\
peut inhiber l'action \\
négative de ZA sur le \\
remodelage osseux
\end{tabular} & $\begin{array}{l}\text { - } 9 \text { chiens (Beagle) } \\
\text { - } 3 \text { groupes traités IV par solution } \\
\text { saline (G1) ou 2,5 mg ZA (G2) ou } 5 \text { mg } \\
\text { ZA (G3). } \\
\text { - Extractions dentaires après } 45 \text { jours } \\
\text { - G1 : alvéole vide à droite / éponge } \\
\text { de collagène à gauche } \\
\text { - G2 et G3 : comblement rhBMP-2 + } \\
\text { collagène à droite / collagène seul à } \\
\text { gauche } \\
\text { - Analyse à } \mathrm{J15} \text { ( } 4 \mathrm{~mm} \text { apicaux) }\end{array}$ & $\begin{array}{l}\text { - Dans les groupes traités } \\
\text { par ZA, la cicatrisation est } \\
\text { retardée } \\
\text { - rhBMP-2/collagène per- } \\
\text { met de contrecarrer locale- } \\
\text { ment les effets de ZA } \\
\text { - Volume d'os néoformé } \\
\text { similaire entre alvéole vide } \\
\text { et comblée par collagène } \\
\text { (G1) }\end{array}$ & $\begin{array}{l}\text { Le volume d'os néoformé } \\
\text { est similaire }(p<0,1) \text { dans } \\
\text { les alvéoles traitées avec } \\
\text { les éponges de collagène } \\
\text { par rapport aux alvéoles } \\
\text { laissées vides (mesure à } \\
15 \text { jours, } 4 \mathrm{~mm} \text { apicaux de } \\
\text { l'alvéole) }\end{array}$ \\
\hline $\begin{array}{l}\text { Cardaropoli et al. } \\
\text { [23] }\end{array}$ & $\begin{array}{l}\text { Évaluer l'influence de } \\
\text { différents biomaté- } \\
\text { riaux dans la cicatri- } \\
\text { sation }\end{array}$ & $\begin{array}{l}\text { - } 5 \text { chiens (Bâtards) } \\
\text { - } 3 \text { mois après extraction des prémo- } \\
\text { laires et } 1^{\text {eres }} \text { molaires mandibulaires, } \\
\text { création de } 3 \text { défauts cylindriques } \\
(3,5 \mathrm{~mm} \times 8 \mathrm{~mm}) \text { bilatéraux } \\
\text { - Comblement par : } \\
\text { - Bio-0ss }{ }^{\circledR} / \text { Collagen } \\
\text { - Éponge de collagène } \\
\text { - Vide } \\
\text { Analyse à } 3 \text { mois (histologie) }\end{array}$ & $\begin{array}{l}\text { - Le comblement par } \\
\text { Bio-Oss }{ }^{\circledR} / \text { Collagen et } \\
\text { Collagène limite } \\
\text { l'invagination des tissus } \\
\text { mous et aboutit à un plus } \\
\text { grand volume d'os } \\
\text { minéralisé que dans les } \\
\text { défauts laissés vides } \\
\text { - Aucune analyse } \\
\text { statistique n'a été réalisée }\end{array}$ & $\begin{array}{l}\text { - Il semble utile de placer } \\
\text { une éponge de collagène } \\
\text { dans un défaut pour stabili- } \\
\text { ser le caillot sanguin, limi- } \\
\text { ter la perte de volume } \\
\text { osseux et l'invagination des } \\
\text { tissus mous } \\
\text { - Limites de l'étude : défaut } \\
\text { créé chirurgicalement et } \\
\text { pas d'analyse statistique }\end{array}$ \\
\hline $\begin{array}{l}\text { Mannai et al. } \\
{[24]}\end{array}$ & $\begin{array}{l}\text { Évaluation de la cica- } \\
\text { trisation d'alvéoles } \\
\text { d'extraction après } \\
\text { extraction mise en } \\
\text { place d'éponge de } \\
\text { collagène }\end{array}$ & $\begin{array}{l}\text { - } 4 \text { chiens (Mongrel) } \\
\text { - } 4 \text { dents extraites de façon bilatérale } \\
\text { - Comblement par Collaplug }{ }^{\circledR}(n=2) \\
\text { ou bien laissé vide }(n=2) \\
\text { - Analyse à } 3 \text { jours, } 7 \text { jours ou } 6 \text { mois } \\
\text { (histologie) }\end{array}$ & $\begin{array}{l}\text { Pas de différence de cicatri- } \\
\text { sation entre les échan- } \\
\text { tillons comblés par } \\
\text { collagène et ceux laissés } \\
\text { vides, quel que soit le } \\
\text { temps d'observation }\end{array}$ & $\begin{array}{l}\text { Les éponges de collagène } \\
\text { utilisées dans cette étude } \\
\text { n'ont pas induit de réaction } \\
\text { à corps étranger, mais elles } \\
\text { n'ont pas accéléré la cica- } \\
\text { trisation osseuse }\end{array}$ \\
\hline $\begin{array}{l}\text { Magro-Ernica } \\
\text { et al. } \\
{[26]}\end{array}$ & $\begin{array}{l}\text { Évaluer si la mise en } \\
\text { place d'éponge dans } \\
\text { l'alvéole d'extraction } \\
\text { interfère sur la cica- } \\
\text { trisation osseuse }\end{array}$ & $\begin{array}{l}\text { - } 30 \text { rats albinos } \\
\text { - Extraction de l'incisive centrale } \\
\text { maxillaire droite sur tous les rats } \\
-2 \text { groupes : comblement pas éponge } \\
\text { de collagène ou alvéole laissée vide } \\
-5 \text { animaux par groupe sacrifiés à } 7, \\
21 \text { et } 28 \text { jours (histologie) }\end{array}$ & $\begin{array}{l}\text { Os néoformé à } 7 \text { jours : } \\
\text { - Groupe collagène : } 3,3 \% \\
\text { - Groupe contrôle : } 8,1 \% \\
\text { Os néoformé à } 21 \text { jours : } \\
\text { - Groupe collagène : } 33 \% \\
\text { - Groupe contrôle: } 34,4 \% \\
\text { Os néoformé à } 28 \text { jours : } \\
\text { - Groupe collagène : } 41,3 \% \\
\text { - Groupe contrôle : } 41 \%\end{array}$ & $\begin{array}{l}\text { - Qualité finale de la cica- } \\
\text { trisation comparable dans } \\
\text { les } 2 \text { conditions } \\
\text { - À } 7 \text { jours, moins d'os néo- } \\
\text { formé dans les échan- } \\
\text { tillons comblés avec } \\
\text { éponge de collagène (diffé- } \\
\text { rence significative) } \\
\text { - La mise en place } \\
\text { d'éponges de collagène } \\
\text { retarde la cicatrisation } \\
\text { osseuse précoce }\end{array}$ \\
\hline
\end{tabular}


Tableau I. Suite.

Table I. Continued

\begin{tabular}{|c|c|c|c|c|}
\hline Référence & Objectif de l'etude & Matériel et methodes & Résultats & $\begin{array}{l}\text { Conclusions (sur les } \\
\text { éponges de collagène) }\end{array}$ \\
\hline $\begin{array}{l}\text { Jovanovic et al. } \\
\text { [25] }\end{array}$ & $\begin{array}{l}\text { Évaluer l'efficacité de la } \\
\text { préservation osseuse } \\
\text { post-extractionnelle } \\
\text { obtenue avec des } \\
\text { éponges de collagène } \\
\text { chargées en rhBMP-2, } \\
\text { associée ou non à une } \\
\text { régénération osseuse } \\
\text { guidée }\end{array}$ & $\begin{array}{l}\text { - } 7 \text { chiens Hound } \\
\text { - Défauts mandibulaires créés } \\
\text { chirurgicalement } \\
4 \text { groupes : } \\
\text { - rhBMP-2/éponge de collagène } \\
\text { - rhBMP-2/éponge de collagène/ } \\
\text { ROG } \\
\text { - ROG avec ou sans éponge de } \\
\text { collagène } \\
\text { - Témoins (vide ou éponge de } \\
\text { collagène) } \\
\text { - Analyse à } 12 \text { semaines }\end{array}$ & $\begin{array}{l}\text { - Une reconstruction osseuse } \\
\text { complète a été observée dans } \\
\text { les défauts osseux comblés } \\
\text { avec rhBMP-2 + collagène } \\
\text { - Dans les groupes contrôles, } \\
\text { aucune différence n'a été } \\
\text { observée entre les défauts vides } \\
\text { et les défauts comblés avec } \\
\text { éponge de collagène }\end{array}$ & $\begin{array}{l}\text { Dans les groupes } \\
\text { contrôles, il n'a pas été } \\
\text { observé de différence } \\
\text { entre les défauts osseux } \\
\text { laissés vides et ceux com- } \\
\text { blés avec une éponge de } \\
\text { collagène }\end{array}$ \\
\hline $\begin{array}{l}\text { De Sarkar } \\
\text { [28] }\end{array}$ & $\begin{array}{l}\text { Évaluation de l'effet } \\
\text { d'une délivrance locale } \\
\text { d'alendronate après } \\
\text { extraction de dent de } \\
\text { sagesse pour prévenir la } \\
\text { résorption osseuse } \\
\text { alvéolaire } \\
\text { post-extractionnelle }\end{array}$ & $\begin{array}{l}\text { - } 20 \text { patients } \\
\text { - Extraction de } 4 \text { dents de } \\
\text { sagesse } \\
2 \text { groupes : } \\
\text { - Vide vs. éponge de collagène } \\
\text { - Alendronate sur éponge de } \\
\text { collagène vs. éponge de } \\
\text { collagène seule } \\
\text { - Suivi clinique et radiologique }\end{array}$ & $\begin{array}{l}1 \text { mois post op: } \\
\text { Résorption osseuse inférieure } \\
\text { dans le groupe comblé avec } \\
\text { collagène }(p<0,004) \\
4 \text { mois post-op: } \\
\text { - Collagène seul : } 77,2 \% \text { de la } \\
\text { résorption osseuse observée } \\
\text { dans le témoin vide } \\
\text { - Collagène + alendronate : } 55, \\
62 \% \text { de la résorption osseuse } \\
\text { observée dans le témoin vide } \\
\text { ( } p<0,001)\end{array}$ & $\begin{array}{l}\text { Après } 4 \text { mois de cicatrisa- } \\
\text { tion, la mise en place } \\
\text { d'éponges de collagène } \\
\text { semble limiter la résorp- } \\
\text { tion osseuse par rapport à } \\
\text { une alvéole laissée vide } \\
\text { (pas de valeur statistique } \\
\text { donnée) }\end{array}$ \\
\hline $\begin{array}{l}\text { Fiorellini et al. } \\
2005 \\
{[27]}\end{array}$ & $\begin{array}{l}\text { Évaluer l'efficacité de la } \\
\text { préservation osseuse } \\
\text { post-extractionnelle } \\
\text { obtenue avec des } \\
\text { éponges de collagène } \\
\text { chargées en rhBMP-2 } \\
\text { (étude clinique) }\end{array}$ & $\begin{array}{l}\text { - } 80 \text { patients répartis en } \\
4 \text { groupes : } \\
\quad \text { - rhBMP-2 } 0,75 \mathrm{mg} / \mathrm{ml}(n=21) \\
\text { - rhBMP-2 } 1,50 \mathrm{mg} / \mathrm{ml}(\mathrm{n}=22) \\
\text { - Éponge de collagène seule } \\
(\mathrm{n}=17) \\
\quad \text { - Alvéole vide }(\mathrm{n}=20) \\
\text { - Quantification os néoformé sur } \\
\text { scanner à } 16 \text { semaines }\end{array}$ & $\begin{array}{l}\text { - Augmentation significative du } \\
\text { volume d'os néoformé avec } \\
\text { rhBMP-2 } \\
\text { - Pas de différence entre groupe } \\
\text { traité par éponge de collagène } \\
\text { seule et alvéoles laissées vides }\end{array}$ & $\begin{array}{l}\text { La mise en place d'une } \\
\text { éponge de collagène dans } \\
\text { l'alvéole dentaire ne per- } \\
\text { met pas d'améliorer la } \\
\text { cicatrisation osseuse post- } \\
\text { extractionnelle chez } \\
\text { l'homme }\end{array}$ \\
\hline
\end{tabular}

collagène dans une alvéole pour limiter la résorption, en comparaison de la cicatrisation osseuse naturelle [27].

\section{Utilisation des éponges de collagène pour la régénération osseuse extra-orale}

Quatre études précliniques utilisant le collagène pour reconstruire des défauts osseux extra-oraux créés chirurgicalement ont été incluses dans cette revue systématique.

Parmi les quatre études, il y avait 1 modèle chez le chien et 3 chez le rat (Tab. II). Dans un cas, il s'agissait de défauts osseux au niveau de la crête iliaque [20]. Dans deux cas, il s'agissait de défaut osseux de calvaria $[29,30]$ et dans un autre cas il s'agissait d'une perforation de l'os du fémur [31].

Dans les études sélectionnées, la néoformation osseuse après comblement avec une éponge de collagène a été comparée avec des défauts laissés vides [20,29-31], d'autres agents hémostatiques [20], du ßTCP, du Bio0ss Collagen ${ }^{\circledR}$ [29] ou encore un gel d'acide hyaluronique [30].

Dans une étude, les défauts comblés avec une éponge de collagène montrent un volume d'os néoformé significativement supérieur aux défauts laissés vides [31]. Cependant, ce n'est pas ce que montrent les coupes histologiques où les échantillons témoins sans comblement présentent une cicatrisation osseuse supérieure après 28 jours de cicatrisation. De plus, les résultats 
Tableau II. Effet des éponges de collagène sur la régénération osseuse extra-orale $(\mathrm{AH}$ : acide hyaluronique ; $F C-H A C$ : collagène fibrillaire dénaturé thermiquement; TCP : phosphate tricalcique).

Table II. Effect of acellular collagen sponges on extra-oral bone regeneration (AH: hyaluronic acid; FC-HAC: fibrillar and heat-denatured collagen; TCP: tricalcium phosphate).

\begin{tabular}{|c|c|c|c|c|}
\hline Référence & Objectif de l'étude & Matériel et méthodes & Résultats & $\begin{array}{l}\text { Conclusions (sur les } \\
\text { éponges de collagène) }\end{array}$ \\
\hline $\begin{array}{l}\text { Finn et al. } \\
{[20]}\end{array}$ & $\begin{array}{l}\text { Évaluation de } \\
\text { l'influence de } 4 \text { agents } \\
\text { hémostatiques sur la } \\
\text { cicatrisation osseuse } \\
\text { dans un site de } \\
\text { prélèvement osseux } \\
\text { (crête illiaque) }\end{array}$ & $\begin{array}{l}\text { - } 4 \text { chiens } \\
\text { - } 5 \text { défauts par crête illiaque } \\
\text { - Comblements par: Avitene }{ }^{\circledR} \text { (Collagène), } \\
\text { Bone wax }{ }^{\circledR}, \text { Gelfoam }{ }^{\circledR} \text { (gélatine), } \\
\text { Surgicel }{ }^{\circledR}(\text { cellulose), vide } \\
\text { - Sacrifice à } 2 \text { mois (suivi radiographique et } \\
\text { histologique) }\end{array}$ & $\begin{array}{l}\text { - Néoformation osseuse } \\
\text { similaire dans toutes les } \\
\text { conditions ; présence de } \\
\text { matériau résiduel avec } \\
\text { Avitene }^{\circledR} \text { et Gelfoam }{ }^{\circledR} \\
\text { sans réaction à corps } \\
\text { étranger } \\
\text { - Pas de quantification }\end{array}$ & $\begin{array}{l}\text { - Avitène }{ }^{\circledR} \text { et Gelfoam }{ }^{\circledR} \\
\text { n'ont pas d'influence } \\
\text { négative ni positive sur la } \\
\text { néoformation osseuse en } \\
\text { comparaison à un défaut } \\
\text { témoin laissé vide }\end{array}$ \\
\hline $\begin{array}{l}\text { Zigdon et al. } \\
\text { [29] }\end{array}$ & $\begin{array}{l}\text { Évaluer le potentiel de } \\
\text { régénération de } \\
\text { plusieurs biomatériaux } \\
\text { pour l'augmentation } \\
\text { osseuse verticale sur la } \\
\text { calvaria de rat }\end{array}$ & $\begin{array}{l}\text { - } 16 \text { rats } \\
\text { - Mise en place de dômes en or ménageant un } \\
\text { espace pour la régénération osseuse verticale } \\
\text { sur la calvaria } \\
\text { - Comblement avec BioOss Collagen }{ }^{\circledR}, \beta T C P \text {, } \\
\text { Éponge de Collagène, témoin vide } \\
\text { - Histologie à } 8 \text { semaines }\end{array}$ & $\begin{array}{l}\text { - Augmentation osseuse } \\
\text { verticale est } \\
\text { significativement } \\
\text { supérieure avec BTCP } \\
\text { - Témoin vide et groupe } \\
\text { "éponges de collagène" } \\
\text { aboutissent à une } \\
\text { régénération osseuse } \\
\text { verticale limitée et } \\
\text { similaire entre ces } \\
2 \text { groupes }\end{array}$ & $\begin{array}{l}\text { La mise en place d'éponge } \\
\text { de collagène ne permet } \\
\text { pas un gain osseux } \\
\text { vertical significatif } \\
\text { comparé au contrôle vide }\end{array}$ \\
\hline $\begin{array}{l}\text { De Brito } \\
\text { Bezerra } \\
{[30]}\end{array}$ & $\begin{array}{l}\text { Tester un gel d'AH pour } \\
\text { la régénération osseuse }\end{array}$ & $\begin{array}{l}32 \text { rats Wistar } \\
\text { Défauts osseux de calvaria } \\
\text { Groupes : } \\
\text { - Gel AH } \\
\text { - Gel AH + éponge de collagène } \\
\text { - Vide } \\
\text { - Collagène } \\
\text { - Analyse à } 60 \text { jours (histologie) }\end{array}$ & $\begin{array}{l}\text { - Régénération osseuse } \\
\text { incomplète dans tous les } \\
\text { cas } \\
\text { - Régénération osseuse } \\
\text { AH+collagène supérieure } \\
\text { aux autres conditions } \\
\text { - Pas de différence entre } \\
\text { défauts vides et comblés } \\
\text { par éponge de collagène }\end{array}$ & $\begin{array}{l}\text { L'implantation d'éponges } \\
\text { de collagène dans un } \\
\text { défaut osseux de calvaria } \\
\text { de rat ne permet pas de } \\
\text { régénération osseuse } \\
\text { supérieure aux défauts } \\
\text { laissés vides }\end{array}$ \\
\hline $\begin{array}{l}\text { Shimoji et al. } \\
\text { [31] }\end{array}$ & $\begin{array}{l}\text { Évaluer l'efficacité } \\
\text { d'une éponge de } \\
\text { collagène placée en } \\
\text { onlay à réparer une } \\
\text { perforation osseuse }\end{array}$ & $\begin{array}{l}\text { - } 80 \text { rats Wistar (160 fémurs) } \\
\text { - Incision longitudinale sur la partie } \\
\text { antérolatérale du fémur } \\
-4 \text { groupes : } \\
\text { Perforation + éponge de collagène (PS) } \\
\text { Éponge de collagène seule, sans perforation (S) } \\
\text { Perforation non traitée (P) } \\
\text { Contrôle : pas de perforation ni d'éponge de } \\
\text { collagène (C) } \\
\text { Des rats ont été sacrifiés à } 3,5,10,14 \text { et } \\
28 \text { jours }\end{array}$ & $\begin{array}{l}\text { - Régénération osseuse } \\
\text { sans différences } \\
\text { significatives entre les } \\
\text { groupes PS et } P \text { d'après } \\
\text { résultats histologiques }\end{array}$ & $\begin{array}{l}\text { L'utilisation d'une } \\
\text { FC-HAC n'améliore pas la } \\
\text { cicatrisation osseuse de } \\
\text { façon significative }\end{array}$ \\
\hline
\end{tabular}

de cette étude semblent inconstants car dans la condition contrôle, sans perforation ni éponge, une augmentation du volume osseux est observée après 28 jours de cicatrisation.

En conclusion, la majorité des études ne montrent pas d'amélioration significative de la régénération osseuse en présence d'une éponge de collagène comparée à des défauts laissés vides.

\section{Utilisation des éponges de collagène comme vecteur de biomolécules pour la régénération osseuse et parodontale}

Huit études utilisant le collagène comme vecteur de biomolécules ont été retenues. Il y avait 7 études précliniques et une étude clinique (Tab. III). 
Tableau III. Utilisation d'éponge de collagène vectrice de facteurs de croissance pour la régénération alvéolaire et parodontale (ACS : éponge de collagène acellulaire; BMP : protéine morphogénétique osseuse ; BSM : pâte de phosphate de calcium ; GBR : régénération osseuse guidée ; GDF : facteur de croissance et de différentiation osseuse ; ZA : acide zolédronique).

Table III. Use of collagen sponges as a delivery system of growth and differentiation factors for alveolar bone and periodontal regeneration. ACS : Acellular Collagen Sponge; BMP: Bone Morphogénétic Protein; BSM: Calcium Phosphate Putty; GBR: Guided Bone Regeneration; GDF: Growth and Differentiation Factor; ZA: Zoledronic Acid).

\begin{tabular}{|c|c|c|c|c|}
\hline Référence & Objectif de l'étude & Matériel et méthodes & Résultats & $\begin{array}{l}\text { Conclusion (sur les } \\
\text { éponges de collagène) }\end{array}$ \\
\hline $\begin{array}{l}\text { Jovanovic et al. } \\
\text { [25] }\end{array}$ & $\begin{array}{l}\text { Évaluer la formation d'os } \\
\text { suivant l'implantation de } \\
\text { rhBMP-2/ACS avec ou } \\
\text { sans GBR pour l'augmen- } \\
\text { tation alvéolaire }\end{array}$ & $\begin{array}{l}\text { - } 7 \text { chiens (Hound) } \\
\text { - Défauts mandibulaires créés } \\
\text { chirurgicalement } \\
5 \text { groupes : } \\
\text { - rhBMP-2/ACS } \\
\text { - rhBMP-2/ACS/GBR } \\
\text { - Collagène } \\
\text { - Éponge de Collagène + GBR } \\
\text { - Vide } \\
\text { - Analyse à } 12 \text { semaines }\end{array}$ & $\begin{array}{l}\text { - Les sites avec mise en place } \\
\text { de rhBMP-2/ACS }(0,2 \mathrm{mg} / \mathrm{ml}) \\
\text { présentaient un comblement } \\
\text { osseux d'environ } 101 \% \\
\text { contre } 60 \% \text { pour les sites avec } \\
\text { ACS seule } \\
\text { - La densité osseuse était } \\
\text { similaire pour les } 2 \text { groupes } \\
\text { Site avec échec de cicatrisa- } \\
\text { tion exclu de l'analyse } \\
\text { - Aucunes différences dans les } \\
\text { groupes contrôles entre } \\
\text { défaut vide et défaut comblé } \\
\text { avec éponge de collagène }\end{array}$ & $\begin{array}{l}\text { rhBMP-2/ACS présente un } \\
\text { potentiel significatif pour } \\
\text { reconstruire des défauts } \\
\text { importants de crête osseuse } \\
\text { mandibulaire }\end{array}$ \\
\hline $\begin{array}{l}\text { Gerard et al. } \\
\text { [22] }\end{array}$ & $\begin{array}{l}\text { - Déterminer l 'effet d'une } \\
\text { dose de ZA sur la cicatri- } \\
\text { sation osseuse d'une } \\
\text { alvéole après extraction. } \\
\text { - Évaluer si l'association } \\
\text { rhBMP-2/ACS peut inhiber } \\
\text { l'action de ZA sur le remo- } \\
\text { delage osseux }\end{array}$ & $\begin{array}{l}3 \text { groupes : reçoivent } 15 \mathrm{ml} \text { de } \\
\text { solution saline, } 2,5 \mathrm{mg} \text { de } \mathrm{ZA} \\
\text { ou } 5 \mathrm{mg} \text { de } Z \mathrm{~A} \\
45 \text { jrs après on extrait } 2^{\mathrm{e}} \mathrm{PM} \text { et } \\
1^{\text {ère }} \mathrm{M} \text { droite et gauche sur } \\
9 \text { chiens } \\
\text { Gpe } 1: \text { à droite : cicatrisation } \\
\text { naturelle, à gauche : ACS } \\
\text { Gpe } 2 \text { et } 3: \text { à droite } \\
\text { rhBMP-2/ACS ; à gauche ACS } \\
\text { seule } \\
\text { Analyse histométrique } 15 \text { jours } \\
\text { après extraction }\end{array}$ & $\begin{array}{l}\text { - Biais car injection de ZA } \\
\text { Le pourcentage de volume } \\
\text { osseux dans les } 4 \mathrm{~mm} \text { apicaux } \\
\text { est plus important avec } \\
\text { rhBMP-2/ACS qu'avec ACS } \\
\text { seule } \\
\text { - Groupe rhBMP-2 contient } \\
\text { significativement plus de nou- } \\
\text { vel os formé } \\
\text { - Le taux d'apposition et le } \\
\text { remodelage osseux sont signi- } \\
\text { ficativement plus élevés avec } \\
\text { rhBMP-2/ACS qu'avec ACS } \\
\text { seule }\end{array}$ & $\begin{array}{l}\text { La mise en place de rhBMP- } \\
\text { 2/ACS permet d'augmenter } \\
\text { significativement le remode- } \\
\text { lage osseux et le taux } \\
\text { d'apposition dans les } \\
\text { alvéoles d'extraction }\end{array}$ \\
\hline $\begin{array}{l}\text { Wikesjo et al. } \\
\text { [34] }\end{array}$ & $\begin{array}{l}\text { Étudier la régénération } \\
\text { osseuse alvéolaire, la for- } \\
\text { mation de cément ainsi } \\
\text { que la résorption radicu- } \\
\text { laire et l'ankylose en pré- } \\
\text { sence de rhBMP-2 }\end{array}$ & $\begin{array}{l}\text { - Défauts parodontaux induits } \\
\text { chirurgicalement sur } 8 \text { chiens } \\
\text { (Beagle) } \\
\text { - Mise en place de rhBMP-2/ } \\
\text { ACS aux concentrations de } \\
0,05 ; 0,10 \text { ou } 0,20 \mathrm{mg} / \mathrm{ml} \text { ou } \\
\text { ACS seule } \\
\text { - Analyse histologique et } \\
\text { histométrique à } 2 \text { mois }\end{array}$ & $\begin{array}{l}\text { - Régénération alvéolaire } \\
\text { importante }(86,96 \text { et } 88 \%) \\
\text { de la hauteur de la lésion avec } \\
\text { les concentrations respectives } \\
\text { suivantes : } 0,05 ; 0,10 \text { et } \\
0,20 \mathrm{mg} / \mathrm{ml} \\
\text { - Au niveau de la lésion } \\
\text { contrôle, on a peu ou pas de } \\
\text { régénération }\end{array}$ & $\begin{array}{l}\text { L'implantation de rhBMP-2/ } \\
\text { ACS permet une régénéra- } \\
\text { tion osseuse significative } \\
\text { supérieure en comparaison } \\
\text { de l'implantation de ACS } \\
\text { seule }\end{array}$ \\
\hline $\begin{array}{l}\text { Chiu et al. } \\
\text { [33] }\end{array}$ & $\begin{array}{l}\text { Étudier la régénération } \\
\text { osseuse alvéolaire, la for- } \\
\text { mation de cément ainsi } \\
\text { que la résorption radicu- } \\
\text { laire et l'ankylose asso- } \\
\text { ciée en présence de BMP6 }\end{array}$ & $\begin{array}{l}\text { - Défauts parodontaux de } 4 \text { à } \\
5 \text { mm induits chirurgicalement } \\
\text { sur } 11 \text { chiens (Beagle) } \\
\text { - Mise en place de BMP6/ACS } \\
\text { aux concentrations de } 0,25 ; \\
1,0 \text { et } 2,0 \mathrm{mg} / \mathrm{ml} \text { ou ACS seule. } \\
\text { - Analyse histologique et } \\
\text { histométrique à } 2 \text { mois }\end{array}$ & $\begin{array}{l}\text { - Il y a significativement plus } \\
\text { de nouvel os formé avec ACS/ } \\
\text { BMP6 que dans le contrôle } \\
\text { - Les sites exposés montrent } \\
\text { moins de formation osseuse } \\
\text { - La plus faible dose } \\
0,25 \mathrm{mg} / \mathrm{ml} \text { apparaît comme } \\
\text { étant la plus efficace }\end{array}$ & $\begin{array}{l}\text { L'association BMP6/ACS per- } \\
\text { met une formation osseuse } \\
\text { plus importante que ACS } \\
\text { seule sans développement } \\
\text { cellulaire anarchique lors de } \\
\text { la cicatrisation }\end{array}$ \\
\hline
\end{tabular}


Tableau III. Suite.

Table III. Continued.

\begin{tabular}{|c|c|c|c|c|}
\hline Référence & Objectif de l'étude & Matériel et méthodes & Résultats & $\begin{array}{l}\text { Conclusion (sur les } \\
\text { éponges de collagène) }\end{array}$ \\
\hline $\begin{array}{l}\text { Kim et al. } \\
\text { [32] }\end{array}$ & $\begin{array}{l}\text { Évaluer la formation de } \\
\text { cément et d'os alvéolaire } \\
\text { suite à l'implantation de } \\
\text { rhGDF5/ACS dans un } \\
\text { défaut parodontal créé } \\
\text { chirurgicalement }\end{array}$ & $\begin{array}{l}\text { - Défauts parodontaux à } 1 \text { mur } \\
\text { induit chirurgicalement sur } \\
15 \text { chiens (Beagle) } \\
\text { - Mise en place rhGDF5/ACS à } \\
1 \mu \mathrm{g}, 20 \mu \mathrm{g} \text { et } 100 \mu \mathrm{g} \text { par } \\
\text { défaut ou ACS seule } \\
\text { - Analyse histologique et } \\
\text { histométrique à } 2 \text { mois }\end{array}$ & $\begin{array}{l}\text { Les sites recevant } 1 \mu \mathrm{g} \text { ou } \\
100 \mu \mathrm{g} \text { présentent un gain } \\
\text { osseux en hauteur significati- } \\
\text { vement plus important que le } \\
\text { contrôle. Gain osseux en sur- } \\
\text { face : pas de différence signi- } \\
\text { ficative entre les groupes }\end{array}$ & $\begin{array}{l}\text { L'association de rhBMP5/ACS } \\
\text { permet un gain en hauteur } \\
\text { plus important que ACS } \\
\text { seule et peut être utilisée } \\
\text { sans complication }\end{array}$ \\
\hline $\begin{array}{l}\text { Choi et al. } \\
\text { [35] }\end{array}$ & $\begin{array}{l}\text { Évaluer la régénération } \\
\text { de l'os alvéolaire, du } \\
\text { cément, l'association de } \\
\text { résorption radiculaire ou } \\
\text { d'ankylose suite à } \\
\text { l'implantation de } \\
\text { rhBMP-2/ACS }\end{array}$ & $\begin{array}{l}\text { - Sur } 8 \text { chiens, création de } \\
\text { défauts parodontaux à } 3 \text { murs } \\
\text { au niveau prémolaire maxil- } \\
\text { laire et mandibulaire } \\
\text { - } 3 \text { groupes : rhBMP-2/ACS ; } \\
\text { ACS ; contrôle (laissé vide) } \\
\text { - Analyse radiographique et } \\
\text { histomorphométrique à } 8,16 \\
\text { et } 24 \text { semaines }\end{array}$ & $\begin{array}{l}\text { - Analyse radio : régénération } \\
\text { alvéolaire significativement } \\
\text { plus élevée avec rhBMP-2/ACS } \\
\text { à } 8,16 \text { et } 24 \text { semaines que le } \\
\text { contrôle et plus élevée à } \\
8 \text { semaines pour ACS seule } \\
\text { - Analyse histométrique : le } \\
\text { volume d'os néoformé est de } \\
65 \text { et } 68 \% \text { à } 8 \text { et } 24 \text { semaines } \\
\text { dans les défauts recevant } \\
\text { rhBMP-2 contre } 52 \text { et } 53 \% \\
\text { avec ACS seule }\end{array}$ & $\begin{array}{l}\text { L'évaluation radiologique, } \\
\text { histologique et } \\
\text { histomorphométrique } \\
\text { montre une meilleure } \\
\text { formation osseuse dans les } \\
\text { défauts recevant rhBMP-2/ } \\
\text { ACS que ceux recevant ACS } \\
\text { seule ou ceux laissés vides }\end{array}$ \\
\hline $\begin{array}{l}\text { Blumenthal } \\
\text { et al. } \\
\text { [36] }\end{array}$ & $\begin{array}{l}\text { Évaluer la régénération } \\
\text { de l'os alvéolaire, du } \\
\text { cément, l'association de } \\
\text { résorption radiculaire ou } \\
\text { d'ankylose suite à } \\
\text { l'implantation de } \\
\text { rhBMP-2/ACS ou de } \\
\text { rhBMP-2/ } \alpha B S M\end{array}$ & $\begin{array}{l}\text { - Sur } 4 \text { singes, création de } \\
\text { défauts parodontaux au niveau } \\
\text { maxillaire et mandibulaire } \\
\text { - } 4 \text { groupes : rhBMP-2/ACS ; } \\
\text { rhBMP-2/ } \alpha B S M ; A C S ; \alpha B S M \\
\text { - Analyse histologique et his- } \\
\text { tométrique à } 4 \text { mois }\end{array}$ & $\begin{array}{l}\text { - Les défauts recevant } \\
\text { rhBMP-2/ACS et rhBMP-2/ } \\
\alpha B S M \text { montrent une meilleure } \\
\text { régénération que les contrôles } \\
\text { - Pas de différence entre } \\
\text { rhBMP-2/ACS et rhBMP-2/ } \\
\text { aBSM en ce qui concerne la } \\
\text { néoformation osseuse }\end{array}$ & $\begin{array}{l}\text { L'apport de rhBMP-2 permet } \\
\text { d'améliorer } \\
\text { significativement la } \\
\text { régénération alvéolaire }\end{array}$ \\
\hline $\begin{array}{l}\text { Fiorellini et al. } \\
\text { [27] }\end{array}$ & $\begin{array}{l}\text { Évaluer l'efficacité de } \\
\text { l'induction osseuse avec } \\
\text { deux concentrations de } \\
\text { rhBMP-2, une éponge de } \\
\text { collagène seule et une } \\
\text { alvéole vide après une } \\
\text { extraction dentaire }\end{array}$ & $\begin{array}{l}\text { - Étude sur } 80 \text { patients après } \\
\text { extraction } \\
-4 \text { groupes : } \\
\text { - rhBMP-2 } \\
0,75 \mathrm{mg} / \mathrm{ml}(\mathrm{n}=21) \\
-\mathrm{rhBMP}-2 \\
1,50 \mathrm{mg} / \mathrm{ml}(\mathrm{n}=22) \\
\text { - ACS seul }(\mathrm{n}=17) \\
\text { - pas de traitement }(\mathrm{n}=20) \\
\text { - Analyse scanner à } 16 \text { semaines }\end{array}$ & $\begin{array}{l}\text { - Hauteur du nouvel os signifi- } \\
\text { cativement plus élevé avec } \\
\text { rhBMP-2 que sans. Elle est } \\
\text { significativement plus impor- } \\
\text { tante avec une concentration } \\
\text { de } 1,5 \mathrm{mg} / \mathrm{ml} \text { qu'avec } \\
0,75 \mathrm{mg} / \mathrm{ml} \\
- \text { Maintien du mur palatin de } \\
\text { l'alvéole avec rhBMP-2 à } \\
1,5 \mathrm{mg} / \mathrm{ml}\end{array}$ & $\begin{array}{l}\text { - rhBMP-2/ACS à } 1,5 \mathrm{mg} / \mathrm{ml} \\
\text { semble être la concentration } \\
\text { la plus efficace. } \\
\text { - La présence de rhBMP-2 } \\
\text { induit une formation } \\
\text { osseuse significativement } \\
\text { plus importante qu'avec ACS } \\
\text { seule }\end{array}$ \\
\hline
\end{tabular}

Concernant les 7 études précliniques, il y avait six modèles canins $[22,25,32-35]$ et un modèle chez le primate [36]. Six études concernaient l'utilisation d'éponges de collagène implantées seules ou en association avec des facteurs de croissance dans la régénération de défauts osseux créés chirurgicalement [25,32-36]. Dans trois études, la régénération alvéolaire était évaluée en fonction de différentes concentrations de rhBMP-2 [34], de BMP6 [33], ou de rhGDF5 [32], avec une éponge de collagène comme vecteur. Dans chacune de ces trois études, l'éponge de collagène seule servait de contrôle.
Une autre étude comparait la régénération alvéolaire en présence de rhBMP-2/ACS avec une concentration de $0,2 \mathrm{mg} / \mathrm{ml}$, d'une ACS seule ou d'un défaut parodontal laissé vide [35]. Enfin, une étude évaluait la régénération de l'os alvéolaire d'un défaut parodontal en présence de rhBMP-2/ACS, rhBMP-2/PCa, $A C S$, et $P C a$ [36]. Le phosphate de calcium (PCa) était synthétique $\alpha B S M$ et il servait de vecteur au même titre que les éponges de collagène dans cette étude. Il n'y avait pas de différence significative entre les deux vecteurs de rhBMP-2 en ce qui concerne la néoformation osseuse. 
Deux études précliniques montraient l'utilisation de rhBMP-2 dans la régénération osseuse alvéolaire mandibulaire (Tab. III). L'étude citée précédemment concernant l'effet de l'acide zolédronique sur la cicatrisation osseuse a été retenue dans cette partie car elle permettait d'analyser l'effet de rhBMP-2/ACS sur la cicatrisation par rapport à une éponge de collagène seule et un contrôle laissé vide [22]. Une étude évaluait la formation osseuse avec rhBMP-2 associé à une éponge de collagène dans les défauts importants de crête mandibulaire, avec ou sans GBR, et une ACS seule comme contrôle [25].

En conclusion, dans les cinq études sur la régénération alvéolaire de défauts parodontaux, toutes ont montré une amélioration significative de la régénération osseuse en présence d'une éponge de collagène vecteur d'un facteur de croissance plutôt qu'avec une ACS seule [32-34,36].

Les deux études précliniques sur la régénération osseuse alvéolaire mandibulaire montrent que l'implantation de rhBMP-2 associé à une éponge de collagène est une solution significativement plus efficace qu'une ACS seule pour limiter la résorption post-extractionnelle et corriger des défauts osseux localisés [22,25].

Dans une étude clinique, Fiorellini et al. ont évalué l'efficacité de l'induction osseuse avec deux concentrations de rhBMP-2 $(1,5 \mathrm{mg} / \mathrm{ml}$ et $0,75 \mathrm{mg} / \mathrm{ml})$ délivrée par une ACS comparée à une éponge de collagène seule ou à une cicatrisation osseuse naturelle. La hauteur de l'os néo-formé était significativement plus élevée avec rhBMP-2/ACS que ACS seule ou que le contrôle vide. La présence de rhBMP-2 a induit une formation osseuse significativement plus importante que ACS seule. La concentration de $1,5 \mathrm{mg} / \mathrm{ml}$ était significativement plus efficace que la dose de $0,75 \mathrm{mg} / \mathrm{mL}$ [27]. La mise en place de rhBMP-2+ACS permet de maintenir la hauteur et la largeur de la crête alvéolaire.

\section{Discussion}

L'objectif principal de cette étude était d'évaluer si la mise en place d'éponges de collagène hémostatiques présentait un intérêt pour préserver le volume d'os alvéolaire avant extraction. Globalement, les résultats observés démontrent qu'il n'y a pas d'intérêt à placer une éponge de collagène dans une alvéole d'extraction pour limiter la résorption osseuse à court ou à long terme.

La méthode de recherche bibliographique a suivi les recommandations du Guide d'analyse de la littérature et gradation des recommandations de l'HAS. On a retenu des études présentant un niveau de preuve parfois faible car peu d'études répondaient à notre problématique. Du fait du manque d'études et de leur hétérogénéité, il était difficile de comparer ces études entre elles. En effet, les méthodes conventionnelles utilisant des xénogreffes osseuses donnent des résultats reproductibles et durables [37]. De plus, les indications d'utilisation des éponges de collagène par les fabricants sont des utilisations à visée hémostatique [14].

On a pu constater que la mise en place d'une éponge de collagène seule ne permet pas de limiter de manière significative la résorption osseuse post-extractionnelle (Tab. I). Ceci également en accord avec les conclusions de Singh et al. qui ont montré que la mise en place d'éponges de gélatine (Gelfoam $\left.{ }^{\circledR}\right)$ dans les alvéoles d'extraction de dents de sagesse ne permettait pas de prévenir la formation d'une déhiscence osseuse en distal de la deuxième molaire [38], en comparaison avec un comblement d'hydroxyapatite (témoin positif).

L'éponge de collagène est un bon moyen d'augmenter le recrutement des cellules pour amorcer la cicatrisation mais il n'est pas durable car la résorption de l'éponge est totale au bout de quelques semaines [14]. D’après Takahashi et al., la résorption de l'éponge de collagène est trop rapide pour permettre de maintenir un espace pour la formation osseuse [21]. L'éponge permet de stabiliser le caillot et de limiter l'invagination des tissus mous à court terme; à plus long terme, le volume de l'éponge diminue et ne permet donc pas un maintien de l'espace alvéolaire [39].

Dans l'étude de Magro-Ernica, on a noté la persistance de l'éponge de collagène 7 jours après implantation, ce qui a entraîné un retard de cicatrisation. En effet à 7 jours, on observait de fines travées osseuses au niveau du tiers apical dans les deux groupes mais il y avait significativement plus d'os néoformé dans le groupe contrôle laissé vide $(8,1 \%)$ que dans le groupe avec l'éponge de collagène $(3,3 \%)$ [26].

A priori, de nombreux auteurs semblent penser qu'il n'y a pas d'effet des éponges de collagène sur la régénération osseuse car ils l'utilisent comme matériau contrôle négatif dans des études de régénération osseuse alvéolaire $[22,25,28,32-34,36]$. De plus, Bui et al. ont montré qu'il y avait environ $4 \%$ de complications post-opératoires (dont $1 / 4$ d'alvéolites) après avulsion lorsque l'alvéole était comblée par une éponge de collagène [40], ce qui est similaire aux complications d'extraction sans comblement.

La résorption des éponges de collagène est trop rapide pour permettre de limiter et prévenir la résorption osseuse alvéolaire seule, mais ce collagène semble être un matériau intéressant pour servir de vecteur de facteurs de croissance. En effet, l'utilisation de facteur de croissance associé à l'éponge de collagène a montré de bons résultats et semble être une alternative crédible à la xénogreffe conventionnelle. Dans ce cas, les éponges de collagène sont imprégnées extemporanément avec un facteur de croissance. Il est cependant difficile de connaître la concentration efficace en facteur de croissance car on retrouve beaucoup de différences entre les études. Cependant une étude expérimentale de Pelaez en 2014 sur le rat montre qu'une fois le seuil ostéo-inductif atteint, il n'y a pas d'augmentation significative de la formation osseuse en augmentant la dose [41]. L'étude clinique de Fiorellini 
montre que $1,5 \mathrm{mg} / \mathrm{ml}$ rhBMP-2/ACS serait la concentration la plus efficace pour la préservation de l'os alvéolaire après une extraction dentaire [27].

On a cependant des limites des éponges de collagène en tant que vecteur de biomolécules : l'éponge de collagène n'est pas adaptée pour les indications de greffe en onlay (augmentation de crête en hauteur) car elle subit trop de compression mais elle apparaît satisfaisante en inlay (alvéoles, augmentation sinusienne) $[42,43]$. Cependant, même en situation d'inlay, les éponges de collagène sont comprimées et produisent moins de volume osseux que souhaité [44]. Une autre étude préclinique indique que ACS/rhBMP-2 devrait être mis en place dans la même séance que l'extraction pour plus d'efficacité [45]. Il semblerait que l'on exploite mieux les capacités de l'éponge de collagène chargée en facteur de croissance en l'associant avec une matrice rigide ou du TCP [46-48].

Un autre défaut que l'on observe est le relargage trop rapide des facteurs de croissance lorsqu'ils sont associés avec des membranes de collagène. Le support doit être capable de fixer les protéines pour que leur libération soit complète et progressive. D'après Seeherman [49], la délivrance de la moitié de la dose absorbée de BMP se fait en 5,65 jours avec une éponge de collagène alors qu'elle est de 12,75 jours avec une matrice en phosphate de calcium.

Cependant l'éponge de collagène est un vecteur intéressant car la préparation de l'éponge est rapide et la concentration en rhBMP-2 assez stable. Selon McKay, il y a environ $95 \%$ de protéines qui sont liées à l'éponge de collagène après 15 min de trempage alors qu'il n'y a pas significativement plus de protéine après 60 minutes de trempage dans la solution de rhBMP-2 [50]. D'après INFUSE ${ }^{\circledR}$ Bone Graft (Medtronic, Memphis, TN, USA), même en cas de compression de l'éponge et donc de perte du volume de liquide entre $21 \%$ à $46 \%$, la quantité de rhBMP-2 retenue dans l'éponge excède encore $90 \%$.

Les perspectives d'amélioration de l'éponge de collagène comme vecteur sont importantes : il serait intéressant de pouvoir allonger le temps de résorption de l'éponge ou de pouvoir modifier sa structure pour permettre un relargage plus lent et régulier dans le temps des facteurs de croissance.

D'autres vecteurs de biomolécules peuvent être utilisés. Dans l'étude préclinique de Blumenthal, l'association ACS+rhBMP-2 et $\alpha B S M+r h B M P-2$ a montré des résultats similaires en ce qui concerne la régénération alvéolaire [36].

L'apport de l'ingénierie tissulaire, via des cellules souches et une éponge de collagène, est une perspective intéressante. Par exemple, dans l'étude de d'Aquino et al., les auteurs ont utilisé un matériau composite fait de cellules souches mésenchymateuses $d^{\prime}$ origine pulpaire induites vers un phénotype ostéoblastique et ensemencées sur une éponge de collagène [51]. Après extraction des dents de sagesse mandibulaires, ce matériau cellularisé inséré dans l'alvéole a permis une régénération osseuse complète des alvéoles déshabitées.

\section{Conclusion}

Cette revue systématique de la littérature a mis en évidence que la mise en place d'éponges de collagène hémostatiques dans les alvéoles après extraction dentaire ne permet pas de prévenir la résorption osseuse post-extractionnelle. De même, la majorité des cas étudiés ne semblent pas montrer une efficacité des éponges de collagène dans la régénération osseuse, que ce soit dans des défauts osseux intra-oraux ou extra-oraux.

Par ailleurs, l'apport de facteurs de croissance par l'intermédiaire d'une éponge de collagène semble être prometteur car les résultats précliniques et cliniques ont montré une vraie limitation de la résorption osseuse.

\section{Conflits d'intérêt : aucun}

\section{Références}

1. Trombelli L, Farina R, Marzola A, Bozzi L, Liljenberg B, Lindhe J. Modeling and remodeling of human extraction sockets. J Clin Periodontol 2008;35:630-639.

2. Nanci A. Ten Cate's Oral Histology: Development, Structure, and Function. Elsevier Health Sciences; 2008:337.

3. Fénelon M, Masson-Regnault E, Catros S. La Cicatrisation Osseuse en Chirurgie Orale. Réal Clin 2016.

4. Araújo MG, Lindhe J. Dimensional ridge alterations following tooth extraction. An experimental study in the dog. J Clin Periodontol 2005;32:212-218.

5. Leblebicioglu B, Salas M, Ort Y, Johnson A, Yildiz V0, Kim D-G, et al. Determinants of alveolar ridge preservation differ by anatomic location. J Clin Periodontol 2013;40:387-395.

6. Tan WL, Wong TLT, Wong MCM, Lang NP. A systematic review of post-extractional alveolar hard and soft tissue dimensional changes in humans. Clin Oral Implants Res 2012;23 Suppl 5:1-21.

7. Oghli AA, Steveling H. Ridge preservation following tooth extraction: a comparison between atraumatic extraction and socket seal surgery. Quintessence Int Berl Ger 1985 2010;41:605-609.

8. Fickl S, Zuhr 0, Wachtel H, Stappert CFJ, Stein JM, Hürzeler MB. Dimensional changes of the alveolar ridge contour after different socket preservation techniques. J Clin Periodontol 2008;35: 906-913.

9. Blanco J, Nuñez V, Aracil L, Muñoz F, Ramos I. Ridge alterations following immediate implant placement in the dog: flap versus flapless surgery. J Clin Periodontol 2008;35:640-648.

10. Weng D, Stock V, Schliephake H. Are socket and ridge preservation techniques at the day of tooth extraction efficient in maintaining the tissues of the alveolar ridge? Eur J Oral Implantol 2011;4 Suppl:59-66.

11. Thalmair T, Fickl S, Schneider D, Hinze M, Wachtel H. Dimensional alterations of extraction sites after different alveolar ridge 
preservation techniques - a volumetric study. J Clin Periodontol 2013;40:721-727.

12. Avila-Ortiz G, Elangovan S, Kramer KWO, Blanchette D, Dawson DV. Effect of alveolar ridge preservation after tooth extraction: a systematic review and meta-analysis. J Dent Res 2014;93:950-958.

13. Jung RE, Philipp A, Annen BM, Signorelli L, Thoma DS, Hämmerle $\mathrm{CHF}$, et al. Radiographic evaluation of different techniques for ridge preservation after tooth extraction: a randomized controlled clinical trial. J Clin Periodontol 2013;40:90-98.

14. Haute Autorité de Santé. Rapport d'évaluation technologique. Hémostatiques chirurgicaux 2011. http://www.hasSantefrportailuploaddocsapplicationpdf201107rapporthemostatiques27052011vdpdf n.d.

15. Ardekian L, Gaspar R, Peled M, Brener B, Laufer D. Does low-dose aspirin therapy complicate oral surgical procedures? J Am Dent Assoc 1939 2000;131:331-335.

16. Gestion péri-opératoire des patients traités par antithrombotiques en chirurgie orale. Recommandations. Médecine Buccale Chir Buccale 2015;21:S5-14.

17. Thoma DS, Sancho-Puchades M, Ettlin DA, Hämmerle CHF, Jung RE. Impact of a collagen matrix on early healing, aesthetics and patient morbidity in oral mucosal wounds - a randomized study in humans. J Clin Periodontol 2012;39:157-165.

18. Yetim I, Ozkan OV, Dervişoglu A, Erzurumlu K, Canbolant E. Effect of gentamicin-absorbed collagen in wound healing in pilonidal sinus surgery: a prospective randomized study. J Int Med Res 2010;38:1029-1033.

19. Agence Française de Sécurité Sanitaire des Produits de Santé. Recommandations. Prescription des antibiotiques en pratique bucco-dentaire 2011. http://ansm.sante.fr/Dossiers/Antibiotiques/Odonto-Stomatologie

20. Finn MD, Schow SR, Schneiderman ED. Osseous regeneration in the presence of four common hemostatic agents. J Oral Maxillofac Surg 1992;50:608-612.

21. Takahashi Y, Marukawa E, Omura K. Application of a new material ( $\beta$-TCP/collagen composites) in extraction socket preservation: an experimental study in dogs. Int J Oral Maxillofac Implants 2013;28:444-452.

22. Gerard DA, Carlson ER, Gotcher JE, Pickett D0. Early inhibitory effects of zoledronic acid in tooth extraction sockets in dogs are negated by recombinant human bone morphogenetic protein. $J$ Oral Maxillofac Surg Off J Am Assoc Oral Maxillofac Surg 2014;72:61-66.

23. Cardaropoli G, Araújo M, Hayacibara R, Sukekava F, Lindhe J. Healing of extraction sockets and surgically produced augmented and non-augmented - defects in the alveolar ridge. An experimental study in the dog. J Clin Periodontol 2005;32: 435-440.

24. Mannai C, Leake D, Pizzoferrato A, Ciapetti G, Sangiorgi C. Histologic evaluation of purified bovine tendon collagen sponge in tooth extraction sites in dogs. Oral Surg Oral Med Oral Pathol 1986;61:315-323.

25. Jovanovic SA, Hunt DR, Bernard GW, Spiekermann H, Wozney JM, Wikesjö UME. Bone reconstruction following implantation of rhBMP-2 and guided bone regeneration in canine alveolar ridge defects. Clin Oral Implants Res 2007;18:224-230.
26. Magro-Ernica N, Magro-Filho 0, Rangel-Garcia I. Histologic study of use of microfibrillar collagen hemostat in rat dental sockets. Braz Dent J 2003;14:12-15.

27. Fiorellini JP, Howell TH, Cochran D, MalmquistJ, Lilly LC, Spagnoli $D$, et al. Randomized study evaluating recombinant human bone morphogenetic protein-2 for extraction socket augmentation. J Periodontol 2005;76:605-613.

28. De Sarkar A, Singhvi N, Shetty JN, Ramakrishna T, Shetye 0, Islam $M$, et al. The Local Effect of Alendronate with Intra-alveolar Collagen Sponges on Post Extraction Alveolar ridge Resorption: A Clinical Trial. J Maxillofac Oral Surg 2015;14:344-356.

29. Zigdon H, Lewinson D, Bick T, Machtei EE. Vertical bone augmentation using different osteoconductive scaffolds combined with barrier domes in the rat calvarium. Clin Implant Dent Relat Res 2014;16:138-144.

30. de Brito Bezerra B, Mendes Brazão MA, de Campos MLG, Casati MZ, Sallum EA, Sallum AW. Association of hyaluronic acid with a collagen scaffold may improve bone healing in critical-size bone defects. Clin Oral Implants Res 2012;23:938-942.

31. Shimoji S, Miyaji H, Sugaya T, Tsuji H, Hongo T, Nakatsuka M, et al. Bone perforation and placement of collagen sponge facilitate bone augmentation. J Periodontol 2009;80:505-511.

32. Kim T-G, Wikesjö UME, Cho K-S, Chai J-K, Pippig SD, Siedler M, et al. Periodontal wound healing/regeneration following implantation of recombinant human growth/differentiation factor-5 (rhGDF-5) in an absorbable collagen sponge carrier into one-wall intrabony defects in dogs: a dose-range study. $\mathrm{J}$ Clin Periodontol 2009;36:589-597.

33. Chiu H-C, Chiang C-Y, Tu H-P, Wikesjö UME, Susin C, Fu E. Effects of bone morphogenetic protein- 6 on periodontal wound healing/ regeneration in supraalveolar periodontal defects in dogs. J Clin Periodontol 2013;40:624-630.

34. Wikesjö UM, Guglielmoni P, Promsudthi A, Cho KS, Trombelli L, Selvig KA, et al. Periodontal repair in dogs: effect of rhBMP-2 concentration on regeneration of alveolar bone and periodontal attachment. J Clin Periodontol 1999;26:392-400.

35. Choi S-H, Kim C-K, Cho K-S, Huh J-S, Sorensen RG, Wozney JM, et al. Effect of recombinant human bone morphogenetic protein-2/absorbable collagen sponge (rhBMP-2/ACS) on healing in 3-wall intrabony defects in dogs. J Periodontol 2002;73: $63-72$.

36. Blumenthal NM, Koh-Kunst G, Alves MEAF, Miranda D, Sorensen RG, Wozney JM, et al. Effect of surgical implantation of recombinant human bone morphogenetic protein-2 in a bioabsorbable collagen sponge or calcium phosphate putty carrier in intrabony periodontal defects in the baboon. J Periodontol 2002;73:1494-1506.

37. Aghaloo TL, Moy PK. Which hard tissue augmentation techniques are the most successful in furnishing bony support for implant placement? Int J Oral Maxillofac Implants 2007;22 Suppl:49-70.

38. Singh M, Bhate K, Kulkarni D, Santhosh Kumar SN, Kathariya R. The effect of alloplastic bone graft and absorbable gelatin sponge in prevention of periodontal defects on the distal aspect of mandibular second molars, after surgical removal of impacted mandibular third molar: a comparative prospective study. J Maxillofac Oral Surg 2015;14:101-106. 
39. Pelissier A, Arnault N, Pelissier-Gele B. [Hemorrhage of dental origin. Local hemostatic techniques]. Actual Odonto-Stomatol 1990;44:307-322.

40. Bui CH, Seldin EB, Dodson TB. Types, frequencies, and risk factors for complications after third molar extraction. J Oral Maxillofac Surg 2003;61:1379-89.

41. Pelaez M, Susin C, Lee J, Fiorini T, Bisch FC, Dixon DR, et al. Effect of rhBMP-2 dose on bone formation/maturation in a rat criticalsize calvarial defect model. J Clin Periodontol 2014;41:827-836.

42. Boyne PJ, Lilly LC, Marx RE, Moy PK, Nevins M, Spagnoli DB, et al. De novo bone induction by recombinant human bone morphogenetic protein-2 (rhBMP-2) in maxillary sinus floor augmentation. J Oral Maxillofac Surg 2005;63:1693-1707.

43. Hanisch 0, Tatakis DN, Rohrer MD, Wöhrle PS, Wozney JM, Wikesjö UM. Bone formation and osseointegration stimulated by rhBMP2 following subantral augmentation procedures in nonhuman primates. Int J Oral Maxillofac Implants 1997;12:785-792.

44. Tatakis DN, Koh A, Jin L, Wozney JM, Rohrer MD, Wikesjö UME. Peri-implant bone regeneration using recombinant human bone morphogenetic protein-2 in a canine model: a dose-response study. J Periodontal Res 2002;37:93-100.

45. Hussein KA, Zakhary IE, Hailat D, Elrefai R, Sharawy M, Elsalanty ME. Delayed versus immediate reconstruction of mandibular segmental defects using recombinant human bone morphogenetic protein 2/absorbable collagen sponge. J Oral Maxillofac Surg 2013;71:1107-1118.
46. Wikesjö UME, Qahash M, Thomson RC, Cook AD, Rohrer MD, Wozney JM, et al. Space-providing expanded polytetrafluoroethylene devices define alveolar augmentation at dental implants induced by recombinant human bone morphogenetic protein 2 in an absorbable collagen sponge carrier. Clin Implant Dent Relat Res 2003;5:112-123.

47. Barboza EP, Duarte ME, Geolás L, Sorensen RG, Riedel GE, Wikesjö UM. Ridge augmentation following implantation of recombinant human bone morphogenetic protein-2 in the dog. J Periodontol 2000;71:488-496.

48. Barboza EP, Caúla AL, Caúla F de 0, de Souza R0, Geolás Neto L, Sorensen RG, et al. Effect of recombinant human bone morphogenetic protein-2 in an absorbable collagen sponge with space-providing biomaterials on the augmentation of chronic alveolar ridge defects. J Periodontol 2004;75:702-708.

49. Seeherman H, Wozney JM. Delivery of bone morphogenetic proteins for orthopedic tissue regeneration. Cytokine Growth Factor Rev 2005;16:329-345.

50. McKay WF, Peckham SM, Marotta JS. The Science of RhBMP-2. Quality Medical Pub.; 2006.

51. d'Aquino R, De Rosa A, Lanza V, Tirino V, Laino L, Graziano A, et al. Human mandible bone defect repair by the grafting of dental pulp stem/progenitor cells and collagen sponge biocomplexes. Eur Cell Mater 2009;18:75-83. 